\title{
Spatial Attention: Different Mechanisms for Central and Peripheral Temporal Precues?
}

\author{
Zhong-Lin Lu \\ University of Southern California
}

\author{
Barbara Anne Dosher \\ University of Califormia, Irvine
}

\begin{abstract}
The external noise paradigm (Z.-L. Lu \& B. A. Dosher, 1998) was applied to investigate mechanisms of spatial attention in location precuing. Observers were precued or simultaneously cued to identify 1 of 4 pseudocharacters embedded in various amounts of external noise. The cues were either central or peripheral. Both central and peripheral precuing significantly reduced threshold in the presence of high external noise (16\% and $17.5 \%$ ). Only peripheral precuing significantly reduced threshold in the presence of low, or no, external noise $(11 \%)$. A perceptual template model identified different mechanisms of attention for central and peripheral precuing, external noise exclusion for central precuing, and a combination of external noise exclusion and stimulus enhancement (or equivalently, internal additive noise reduction) for peripheral cuing.
\end{abstract}

In a high workload environment, a human operator might need to monitor a large number of information sources simultaneously. Whereas this might be accomplished by saccades, the eye movement system is limited by the relatively long time (about $200 \mathrm{~ms}$ ) each saccade takes (Hallett, 1986). On the other hand, the existence of a faster spatial attention system was proposed about a century ago (Helmholtz, 1866/1911; Wundt, 1902), selecting regions of the visual field for further processing without eye movements. This view has been established by the more recent literature (e.g., Beck \& Ambler, 1973; Cohn \& Lasley, 1974; Hoffman \& Nelson, 1981; Posner, Snyder, \& Davidson, 1980; Sperling \& Melchner, 1978; Wolford \& Morrison, 1980). However, the mechanisms of spatial attention (how spatial attention improves human performance) remain one of the central questions in cognitive psychology. In this article, we investigate the mechanisms of spatial attention involved in one particular phenomenon: a precuing advantage in multiple-element displays.

\section{Background}

\section{Precuing Advantage}

Many studies in the literature have demonstrated that precuing, that is, informing the observer about the target location before

Zhong-Lin Lu, Laboratory of Brain processES (LOBES), Department of Psychology, University of Southern California; Barbara Anne Dosher, Department of Cognitive Science and Institute of Mathematical Behavioral Sciences, University of California, Irvine.

This research was supported by the Air Force Office of Scientific Research, Life Science Directorate, Visual Information Processing Program.

Correspondence concerning this article should be addressed to Zhong-Lin Lu, Department of Psychology, SGM 501, University of Southern California, Los Angeles, California 90089-1061, or Barbara Anne Dosher, Department of Cognitive Science, SSP3151, University of California, Irvine, California 92697-5100. Electronic mail may be sent to zhonglin@rcf.usc.edu or bdosher@uci.edu. stimulus onset in multiple-element displays, may improve the observer's performance both in terms of accuracy (e.g., Cheal \& Lyon, 1991; Henderson, 1991; Lyon, 1990) and in terms of response time (e.g., Egly \& Homa, 1991; Eriksen \& Hoffman, 1972; Henderson \& Macquistan, 1993). In a typical task (e.g., Henderson, 1991), a target stimulus appears briefly in only one of eight possible locations, all of which are masked by high-contrast patterns immediately after target presentation. For example, in Henderson (1991), 100-ms peripheral temporal precuing of the target location improved two alternative forced choice accuracy by approximately $10 \%$.

\section{Central Versus Peripheral Cuing}

Precues are normally classified as central or peripheral, depending on the spatial location of the cue relative to that of the target stimulus. Central precues point to the target location but are located in the geometric center of all the possible target locations, whereas peripheral precues are located near the target location. There is evidence that central and peripheral cues may activate two different spatial attention systems: an exogenous system and an endogenous system (Briand \& Klein, 1987; Posner, 1980; Posner \& Cohen, 1984). Several functional differences exist between the two systems: reflexive versus voluntary (Jonides \& Yantis, 1988; Nakayama \& Mackeben, 1989), large versus small cuing effects (Henderson, 1991; Jonides, 1981), faster versus slower action (Cheal \& Lyon, 1991; Muller \& Rabbitt, 1989), and differences in inhibition of return (Posner \& Cohen, 1984). In this article, we directly compare the mechanisms of attention in central and peripheral temporal precuing.

\section{Temporal Precuing Versus Cue Validity}

A temporal precuing experiment studies performance differences as a function of cue-target stimulus onset asynchrony (SOA; e.g., Cheal \& Lyon, 1991; Lyon, 1990). A highly related yet different manipulation involves cue validity: A precue is always 
presented at a given cue-target SOA while the validity of the precue is manipulated. In some cases, a simultaneous or delayed report cue ${ }^{1}$ indicates to the observer the target location (Cheal \& Gregory, 1997; Dosher \& Lu, 2000a, 2000b; Downing, 1988; Henderson, 1991, 1996; Posner, 1980; Shiu \& Pashler, 1994). Intrinsically, these two types of experiments may reflect different attention mechanisms because cue validity experiments involve providing the participant with misleading information, whereas temporal precuing experiments do not. In this study, we concentrated on temporal precuing effects. Cue validity effects are studied in another article (Dosher \& Lu, 2000b).

\section{Poststimulus Masking Versus External Noise}

In temporal precuing experiments using accuracy as the dependent measure, poststimulus masks were often used to keep the overall accuracy level in the experiment below ceiling when target stimuli were of high contrast (Cheal \& Lyon, 1992; Henderson, 1996; Lyon, 1990; Shiu \& Pashler, 1994). Some authors (e.g., Cheal \& Lyon, 1992) even identified poststimulus masking as a critical condition for precuing advantages based on comparisons of experiments with and without poststimulus masking or with different forms of poststimulus masking. However, because of the large accuracy differences between different masking conditions, such comparisons are hard to interpret. On the surface, the poststimulus masking procedure is very similar to the external noise plus attention paradigm (Lu \& Dosher, 1998; see below for a description) used in the current studies. In the external noise paradigm, a systematically controlled amount of external noise is combined with the target stimulus. The critical difference between the two procedures is that the external noise plus attention paradigm compares signal contrasts (thresholds) required to produce the same accuracy levels in a range of external noise levels. An additional advantage of performance comparison at threshold is that the threshold regions are the most sensitive (rapidly changing) regions on psychometric functions.

\section{Mechanisms of the Precuing Advantage}

Various mechanisms of spatial attention have been proposed to account for spatial precuing effects in multiple-element displays. The earliest and possibly still the most popular proposal is that spatial precuing facilitates perceptual processing at the cued location (Cheal, Lyon, \& Gottlob, 1994; Corbetta, Miezin, Shulman, \& Petersen, 1993; Mangun, Hillyard, \& Luck, 1993; Posner, Nissen, \& Ogden, 1978). If this were true, spatial precuing would enhance performance in discrimination tasks in the absence of poststimulus masking, a phenomenon that has been debated (Henderson, 1996; Klein \& Hansen, 1990; Shiu \& Pashler, 1994).

Another widely held view is that perceptual processing is resource limited; spatial precuing allocates the limited capacity (Broadbent, 1957, 1971; Henderson, 1996; Henderson \& Macquistan, 1993). Other proposed mechanisms for precuing advantages include elimination of interference from masks or stimulus information in other nontarget locations (Shiu \& Pashler, 1994), suppression of masking at the cued location (Enns \& Di Lollo, 1997), and both facilitation of responses to attended objects and inhibition of responses to other objects (Cheal \& Gregory, 1997).
To summarize, the mechanism of the temporal precuing advantage is still actively debated (Henderson, 1996). Additional testable hypotheses of the proposed mechanisms would be useful in explicating the important functions of attention in these paradigms.

\section{Overview}

In this article, we offer a theoretical framework in which a number of attention mechanisms manifest unique signature performance patterns, as well as an external noise plus attention paradigm (Lu \& Dosher, 1998) that generates critical test data. With the identical procedure and the same observers, we compare mechanisms of attention in central and peripheral temporal precuing by using a task inspired by Lyon (1990). The pattern of performance enables us to identify the mechanism of attentional improvement under the investigated conditions.

\section{Theoretical Framework and the Empirical Paradigm}

In this section, we briefly introduce our theoretical framework and empirical paradigm: the perceptual template model (PTM) and the external noise plus attention paradigm (see Dosher \& Lu, 1999, 2000a; Lu \& Dosher, 1998, 1999b, for more details). In an external noise plus attention paradigm, we studied human performance in a perceptual task in systematically controlled external noise under different attention conditions. Modeling the human perceptual system with a PTM (Figure 1), we generated signature performance patterns for three different mechanisms of attention (Figure 2; Lu \& Dosher, 1998) and derived characteristic data patterns to distinguish mechanism mixtures based on measurements at multiple criterion levels (Figure 3; Dosher \& Lu, 1999, 2000a).

\section{PTM}

Limited by various sources of noise in the perceptual processes (e.g., intrinsic stimulus variability, receptor sampling errors, randomness of neural responses, and loss of information during neural transmission), human perceptual processes exhibit certain inefficiencies. At an overall system level, these inefficiencies can be characterized by using observer models with equivalent internal noise-random internal noise necessary to produce the degree of inefficiency exhibited by the perceptual system (Ahumada \& Watson, 1985; Barlow, 1956; Burgess, Wagner, Jennings, \& Barlow, 1981; Lu \& Dosher, 1999b; Nagaraja, 1964; Pelli, 1981). The amount of equivalent internal noise is normally estimated by systematically manipulating the amount of external noise added to the signal stimulus and observing how threshold - signal stimulus energy required for an observer to maintain a given performance level-depends on the amount of external noise (see Lu \& Dosher, $1999 \mathrm{~b}$, for a review). Although the equivalent noise approach does not distinguish between various sources of observer inefficiency, it does allow us to quantify the overall efficiency of the perceptual system and to compare the efficiency of the perceptual system in

\footnotetext{
${ }^{1}$ Many experiments in the literature use single stimuli and no report cue. The neutral and invalid trials in these experiments suffer from statistical uncertainty loss (see General Discussion). Thus, performance difference between valid and invalid (or neutral) trials may reflect both "attention" effects and stimulus uncertainty losses.
} 

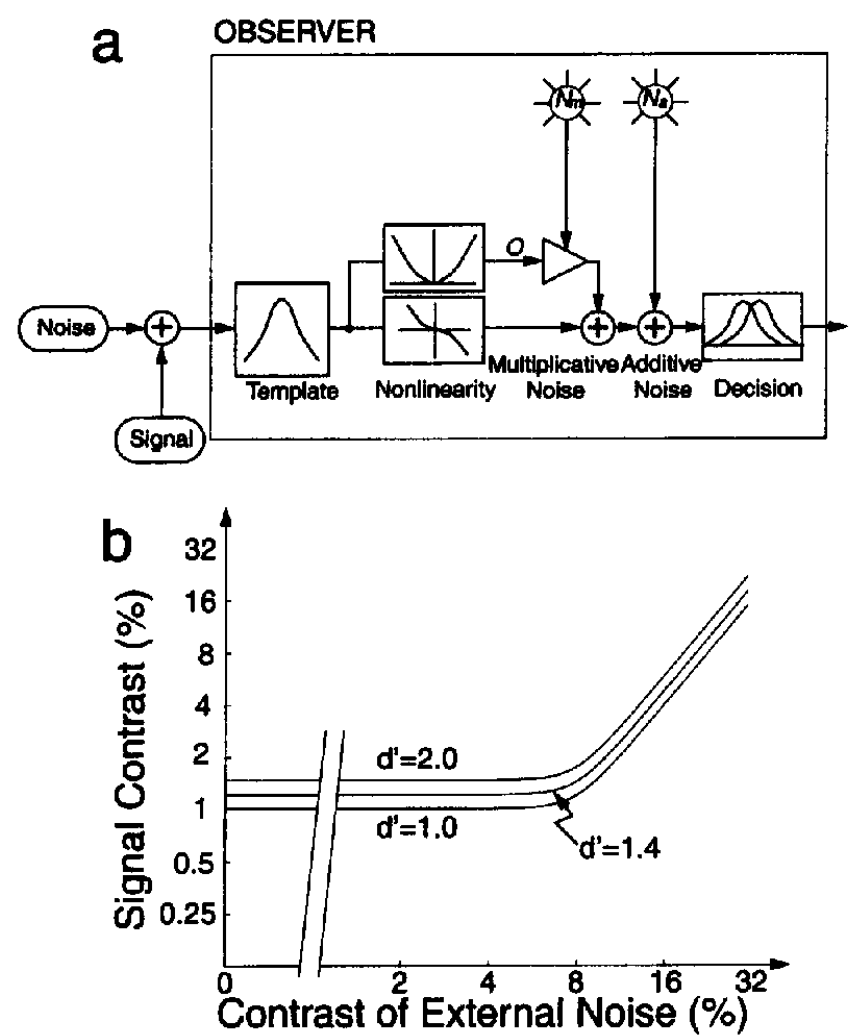

Figure 1. Panel a: A noisy perceptual template model with five major components: (a) a perceptual template; (b) nonlinear transducer function; (c) a multiplicative internal noise source, $N_{\mathrm{m}}$; (d) an additive internal noise source, $N_{\mathrm{g}}$; and (e) a decision process. The triangle denotes an amplifier, which multiplies its two inputs to produce an output. Panel b: Simulated threshold versus external noise contrast functions for a perceptual template model with $N_{\mathrm{m}}=0.2, N_{\mathrm{a}}=0.0039, \beta=4.0$, and $\gamma=2.0$, at three criterion performance levels $\left(d^{\prime}=1.0,1.4\right.$, and 2.0.)

different perceptual tasks (Burgess et al., 1981; Parish \& Sperling, 1991; Pelli, 1981, 1990).

The external noise paradigm and noisy observer models have a long history in visual psychophysics extending over the past half century (see Burgess, Shaw, \& Lubin, 1999, for a review). The PTM model improves and extends many similar models in the literature (Ahumada \& Watson, 1985; Barlow, 1956; Burgess et al., 1981; Nagaraja, 1964; Pelli, 1981). The PTM (Lu \& Dosher, $1998,1999 \mathrm{~b}$ ) is an observer model with five components (Figure 1): (a) A perceptual template (e.g., a spatial frequency filter) with selective tuning. We assume, without losing generality, that the template passes the signal stimulus with gain $\beta$ and the external noise with gain 1.0. (b) A nonlinear transducer function that raises its input to the $\gamma$ power (Foley, 1994; Foley \& Legge, 1981). (c) A multiplicative internal noise with a Gaussian distribution. The mean of the multiplicative noise is 0 ; the standard deviation is proportional (with a coefficient of $N_{\mathrm{m}}$ ) to the total energy in the stimulus. Multiplicative noise is the basis for Weber-law behavior. (d) An independent additive internal noise with a Gaussian distribution. The mean of the additive noise is 0 ; the standard deviation is $N_{\mathrm{a}}$. Additive internal noise is the basis for absolute sensory threshold. (e) A decision process that, depending on the task, could reflect either detection or discrimination and could take the form of either $\mathrm{N}$-alternative forced choice or "yes"/"no" with confidence rating (for details of these models, see MacMillan \& Creelman, 1991).

In a PTM, signal discriminability, $d^{\prime}$, is determined by the strength of the signal, $S$, and the standard deviation of the noise, $\sigma_{N}$, at the decision stage:

$$
d^{\prime}=S / \sigma_{N}
$$

where

$$
S=(\beta c)^{\gamma}
$$

is the strength of the signal (with contrast $c$ ) after the template and the nonlinear transducer; the total variance of the noise, $\sigma_{N}^{2}$ is the sum of the variance of all three (external [ext], multiplicative, and additive) noise sources. ${ }^{2}$

$$
\sigma_{N}^{2}=\sigma_{\mathrm{ext}}^{2 \gamma}+\sigma_{\mathrm{m}}^{2}+\sigma_{\mathrm{a}}^{2}=N_{\mathrm{ext}}^{2 \gamma}+N_{\mathrm{m}}^{2}\left[N_{\mathrm{ext}}^{2 \gamma}+(\beta c)^{2 y}\right]+N_{\mathrm{a}}^{2} .
$$

Combining Equations 1, 2, and 3:

$$
d^{\prime}=\frac{S}{\sigma_{N}}=\frac{(\beta c)^{\gamma}}{\sqrt{N_{\mathrm{ext}}^{2 \gamma}+N_{\mathrm{m}}^{2}\left[(\beta c)^{2 \gamma}+N_{\mathrm{ext}}^{2 \gamma}\right]+N_{\mathrm{n}}^{2}}} .
$$

Thus, for a given performance criterion (i.e., a fixed $d^{\prime}$ ), we can solve Equation 4 to express the threshold contrast $c_{\tau}$ as a function of $N_{\text {ext: }}$

$$
c_{\tau}=\frac{1}{\beta}\left[\frac{\left(1+N_{\mathrm{m}}^{2}\right) N_{\mathrm{ext}}^{2 \gamma}+N_{\mathrm{a}}^{2}}{1 / d^{\prime 2}-N_{\mathrm{m}}^{2}}\right]^{[1 /(2 \gamma)]} .
$$

Figure $1 \mathrm{~b}$ plots threshold versus external noise contrast (TVC) functions in $\log -\log$ form at three performance levels $\left(d^{\prime}=1\right.$, 1.41, and 2) for a PTM model with parameters $\beta=4.0, N_{\mathrm{m}}=0.2$, $N_{\mathrm{a}}=0.0039$, and $\gamma=2.0$. Such graphs possess a characteristic shape: (a) When $N_{\text {ext }} \ll N_{\mathrm{a}}, \log \left(c_{\tau}\right)$ is essentially independent of $N_{\text {ext }}$ because internal additive noise $N_{\mathrm{a}}$ dominates. (b) When $N_{\text {ext }}$ $\gg N_{\mathrm{a}}, \log \left(c_{\mathrm{T}}\right)$ increases as a linear function of $\log \left(N_{\mathrm{ext}}\right)$ because external noise dominates. (c) When $N_{\text {ext }} \approx N_{\mathrm{a}}$, there is a smooth transition from the additive noise dominant region to the external noise dominant region.

\section{Signature Patterns for Three Attention Mechanisms}

In a PTM model, attention improves performance in only three different ways: stimulus enhancement, external noise exclusion, and internal noise reduction. Stimulus enhancement (Lu \& Dosher, 1998; Lu, Liu, \& Dosher, 2000) is modeled by multiplying $N_{\mathrm{a}}$ by a factor of $A_{\mathrm{a}}<1.0$ in the attended condition. Stimulus enhancement and internal additive noise reduction might, in theory, be distinguished biologically but cannot be distinguished on the basis of performance measures. External noise exclusion (Dosher \& Lu, 2000a, 2000b; Lu \& Dosher,

\footnotetext{
${ }^{2}$ Several approximations were made in deriving the total amount of variance. For a discussion of the relationship between the analytical PTM presented here and the stochastic PTM model, see Dosher \& Lu (2000a).
} 
a Stimulus Enhancement

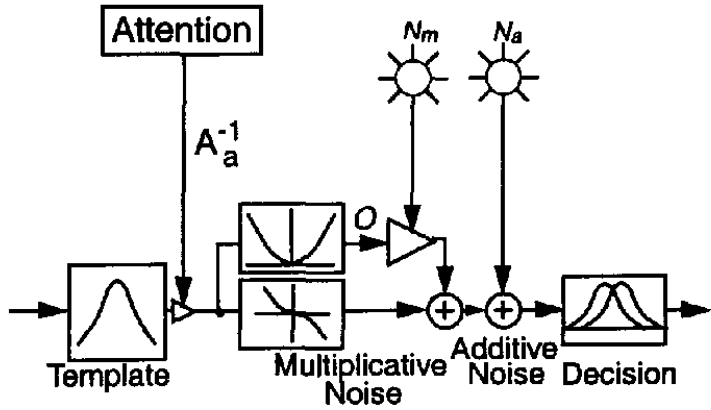

C

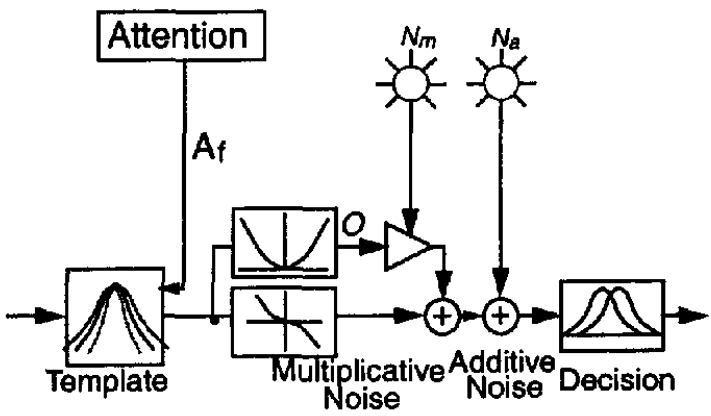

e

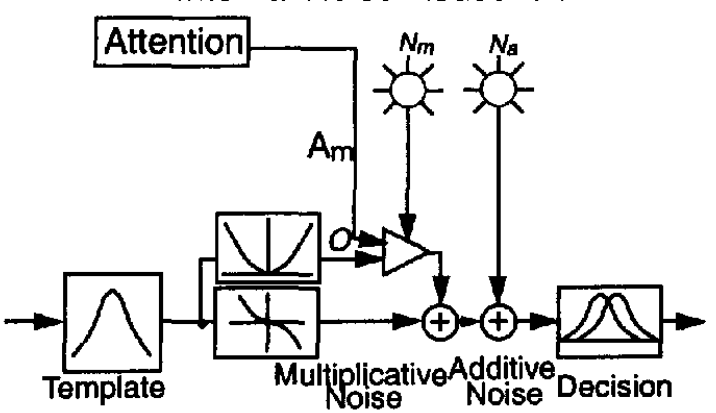

b 100
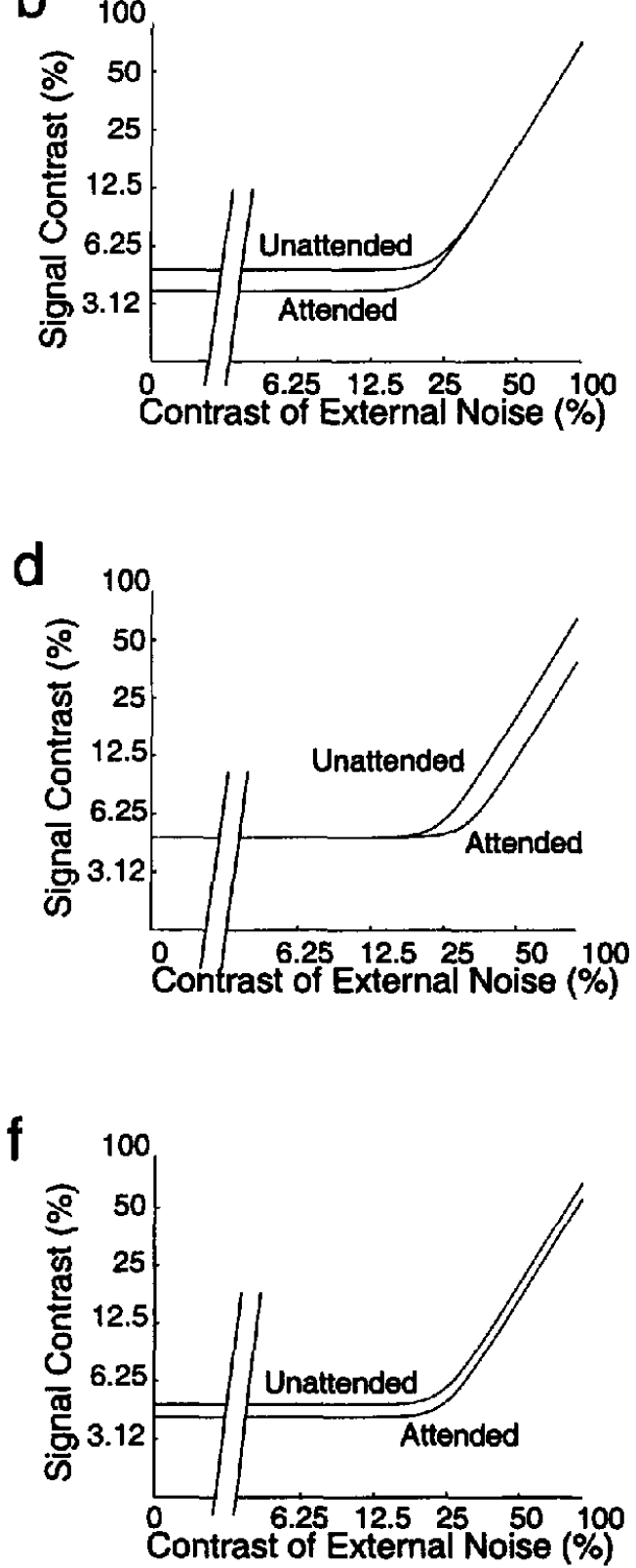

Figure 2. Signature performance patterns for three mechanisms of attention within the framework of a perceptual template model (PTM). Panel a: A PTM in which attention operates by way of stimulus enhancement. Panel b: Signature performance pattern for attention mechanism in Panel a. The threshold versus external noise contrast (TVC) functions in the attended and unattended conditions split at low external noise levels. They overlap with each other at high extemal noise levels. Stimulus enhancernent can improve performance only when the external noise level is low. Panel c: A PTM in which attention operates by way of external noise exclusion. Panel d: Signature performance pattern for attention mechanism in Panel c. For this pair of TVC functions, attention modulates performance only at high levels of external noise. Panel e: A PTM in which attention operates by way of internal multiplicative noise reduction. Panel $f$ : Signature performance pattern for attention mechanism in Panel e. Attention affects performance at all levels of external noise, but increasingly so as external noise increases.

2000), a consequence of better tuning of the perceptual template, is modeled by multiplying $N_{\text {ext }}$ by a factor $A_{\mathrm{f}}<1.0$ in the attended condition. Internal multiplicative noise reduction, a change of the contrast gain control properties of the system, is modeled by multiplying $N_{\mathrm{m}}$ by a factor $A_{\mathrm{m}}<1.0$ in the attended condition. To summarize, in the attended condition, the contrast 
threshold at a given performance level (corresponding to a fixed $d^{\prime}$ ) could potentially be affected by all three mechanisms and is modeled as

$$
c_{\tau}=\frac{1}{\beta}\left[\frac{\left(1+\left(A_{\mathrm{m}} N_{\mathrm{m}}\right)^{2}\right)\left(A_{\mathrm{f}} N_{\mathrm{ext}}\right)^{2 \gamma}+\left(A_{\mathrm{a}} N_{\mathrm{a}}\right)^{2}}{1 / d^{\prime 2}-\left(A_{\mathrm{m}} N_{\mathrm{m}}\right)^{2}}\right]^{[1 /(2 \gamma)]} .
$$

Setting each of the individual $A$ s to be less than 1.0, while leaving the other two $A s$ at 1.0 , generates signature performance patterns for each of the individual attention mechanisms (Figure 2): Stimulus enhancement (or equivalently, internal additive noise reduction) only improves performance at low external noise levels (Figures $2 \mathrm{a}$ and $2 \mathrm{~b}$ ), external noise exclusion only improves performance at high external noise levels (Figures $2 \mathrm{c}$ and $2 \mathrm{~d}$ ), and internal multiplicative noise reduction improves performance at all levels of external noise (Figures $2 \mathrm{e}$ and $2 \mathrm{f}$ ). The mechanism of stimulus enhancement is related to earlier proposals of perceptual facilitation, whereas the mechanism of external noise exclusion is related to proposals of mask suppression.

\section{Distinguish Mechanism Mixtures}

In certain simple situations, a direct comparison of the measured TVC functions and the signature patterns of the PTM model identifies the underlying mechanism of attention (Lu \& Dosher, 1998). In other situations (e.g., observing performance improvement over the entire range of external noise), it is critical to distinguish mechanism mixtures (e.g., a combination of stimulus enhancement and external noise exclusion versus multiplicative noise reduction). A simple solution to this problem is suggested by examining Equation 6: The magnitude of threshold reduction (measured in terms of threshold ratio in the attended and unattended conditions) due to stimulus enhancement $\left(A_{\mathrm{a}}\right)$ or external noise exclusion $\left(A_{f}\right)$ does not depend on $d^{\prime}$, whereas the magnitude of threshold reduction due to multiplicative noise reduction $\left(A_{\mathrm{m}}\right)$ does depend critically on $d^{\prime}$. This is illustrated in Figure 3. Thus, measuring TVC functions at more than two criterion performance levels allows us to compare the magnitude of threshold reduction at different $d^{\prime}$ levels to resolve the individual contribution of each mechanism in an apparent mixture situation (see Dosher \& Lu, 1999, 2000a).

\section{Choice of Experimental Parameters}

The spatial attention task we chose to investigate in this study was inspired by the work of Lyon (1990), who demonstrated large temporal precuing advantages (sensitivity improvements indexed by $d^{\prime}$ ) in a pseudocharacter (rotated $T$ ) identification task in multiple-element displays. In every trial of Lyon's experiment, four pseudocharacters were simultaneously presented at four corners of a square centered around the fixation point for a very brief period, followed by high-contrast masks. Observers were cued (with a dot near the target location) to identify the pseudocharacter at only one location. Lyon found that varying the onset of the cue from $100 \mathrm{~ms}$ prior to the onset of the pseudocharacters to $100 \mathrm{~ms}$ after the onset causes a drop of $d^{\prime}$ from about 2.5 to about 0.5 .

Lyon's (1990) results demonstrated a condition that produces a sizable temporal precuing advantage. On the other hand, the mechanisms of attention underlying the temporal precuing advantage remain debated. To investigate the mechanisms, we applied the
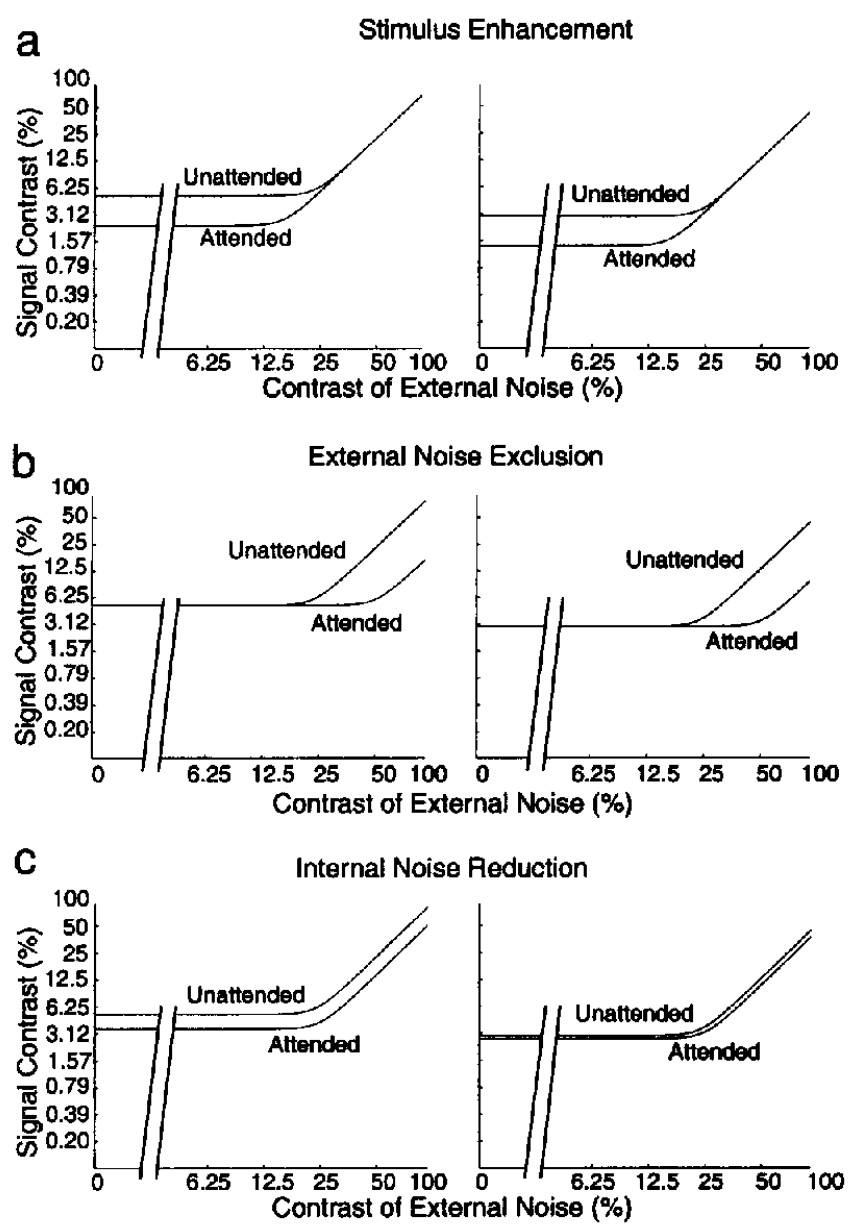

Figure 3. Dependence of the size of the attention effect on performance criteria (following Dosher \& $\mathrm{Lu}, 2000 \mathrm{a}$ ). The panels in the left column plot threshold versus external noise contrast (TVC) functions at $d^{\prime}=0.75$. The panels in the right column plot TVC functions at $d^{\prime}=1.25$ for the same perceptual template model under the same influence of attention. In (a) stimulus enhancement and (b) external noise exclusion, the size of the attention effect is a constant (in a $\log$ scale) across the two performance levels. In (c) multiplicative noise reduction, the size of the attention effect is a highly accelerated increasing function of criterion level $\left(d^{\prime}\right)$.

external noise paradigm and the PTM framework to the basic task used by Lyon under both central and peripheral temporal precuing conditions. Our choice of the specific experimental parameters was based on existing parametric studies of the precuing advantage in the literature. We briefly discuss these factors in turn.

\section{Number of Possible Target Locations}

Others have shown that the size of the precuing advantage increases with the number of possible target locations (Chastain \& Cheal, 1997; Dosher \& Lu, 2000a; Grindley \& Townsend, 1968; Lu \& Dosher, 2000; Shiu \& Pashler, 1994). Typically, one, two, four, or eight possible target locations are used. However, increasing possible target locations to eight might have introduced sensory crowding effects (Dosher \& Lu, 2000a; Lu \& Dosher, 2000; 
Palmer, 1994). We chose to use four possible target locations in this study.

$$
\text { Target Type }
$$

Cheal, Lyon, and Hubbard (1991) and Cheal and Lyon (1992) concluded that the size of the precuing advantage varies in a complex way with target type; the difficulty of task, as gauged by the overall accuracy, could not explain the different magnitude of the temporal precuing advantage over target types. This is consistent with Palmer (1994) who found that, whereas the set-size effects in simple visual search tasks can be fully accounted for by statistical uncertainty effects, the much larger set-size effects in complex visual search tasks can only be explained by a significant loss in perceptual coding in addition to statistical uncertainty effects. The dependence on target type suggests different demands on attentional resources for different perceptual processes. We chose as our task pseudocharacter (rotated $T$ ) discrimination, which has been shown to produce large precuing effects (Cheal et al., 1991; Lyon, 1990).

\section{Cue-Target SOA}

The dependence of the temporal precuing advantage on cuetarget SOAs has been investigated extensively (e.g., Cheal \& Lyon, 1991; Cheal et al., 1991; Lyon, 1990). Peripheral precuing is thought to require only about 100 -ms cue-target SOA to reach its maximum effect. Central precuing is thought to require approximately $300 \mathrm{~ms}$ to achieve its maximum effect in unpracticed observers; in practiced observers, the effect of central precuing at 150-ms SOA is very close to the maximum effect (Cheal \& Lyon, 1991). Because our observers were all well practiced at the two particular SOA conditions (see Method section), we chose a cuetarget SOA of $150 \mathrm{~ms}$ in the precuing conditions. Our choice of the particular SOA reflects our effort to ensure large temporal precuing advantages in both central and peripheral cuing conditions and to avoid saccadic eye movements to the target locations.

\section{Experiment 1: Central Precuing With External Noise}

In this experiment, we chose a task very similar to Lyon (1990) to investigate the mechanisms of attention involved in central precuing advantage. Central cuing was used to isolate the contributions from the endogenous (vs. exogenous) attention system (Briand \& Klein, 1987; Posner, 1980; Posner \& Cohen, 1984). Each of the four spatial locations contained a $T$ in one of four possible orientations, simultaneously presented on the computer screen for a brief period of time. Observers were either precued or simultaneously cued with an arrow in the center of the display to identify the orientation of the T-like pseudocharacter at one of the locations. The pseudocharacters were embedded in systematically varying amount of external noise. The method of constant stimuli was used to measure psychometric functions for pseudocharacter identification for each external noise level in both precuing and simultaneous cuing conditions. Threshold contrasts at three performance criterion levels were calculated for each of the external noise and cuing conditions. PTMs with various combinations of attention mechanisms were fit to the TVC curves at the three criterion performance levels (Dosher \& Lu, 1999) to identify the attention mechanism underlying central or endogenous precue advantage.

\section{Method}

\section{Observers}

Three undergraduate students-AC, SM, and VL-and 2 graduate students-AT and QL-all from the University of Southern California, participated in this experiment. AC, AT, SM, and VL were paid for their participation, and QL volunteered. All observers had normal or correctedto-normal vision. Each observer performed five practice sessions $(2,880$ trials) before the experimental sessions.

\section{Stimuli}

Display apparatus. All stimuli were presented on a Nanao Technology FlexScan-6600 monitor with a P4 phosphor and a refresh rate of 120 frames/s. The display was driven by the internal graphics card in a 7500/100 Power Macintosh, controlled in real time with a program based on a C + + version of Video Toolbox Library (Frederickson, 1996; Pelli \& Zhang, 1991). A special circuit (Pelli \& Zhang, 1991) combined two 8-bit output channels of the video card to produce 6,144 distinct gray levels (12.6 bits). A psychophysical procedure (Lu \& Sperling, 1999) was used to compute the gamma correction for the monitor. The background luminance was set at $27 \mathrm{~cd} / \mathrm{m}^{2}$, with a dynamic range from $1 \mathrm{~cd} / \mathrm{m}^{2}$ to $53 \mathrm{~cd} / \mathrm{m}^{2}$. All displays were viewed binocularly with natural pupil at a viewing distance of approximately $72 \mathrm{~cm}$ in a dimly lighted room.

Pseudocharacters, fixation cross, and visual cues. Four T-like pseudocharacters were created by using $1.15^{\circ} \times 0.14^{\circ}$ line segments that were darker than the background. First, crosses were made of two intersecting line segments, one horizontal and the other vertical. Then, four pseudocharacters, each like a rotated letter $T$, were made by removing each one of the four arms of the crosses. The fixation cross was made of two $0.23^{\circ}$ $\times 0.05^{\circ}$ black line segments. Four arrows, pointing to each of the four stimulus locations, were also made of black line segments. These arrows, always occurring in the center of the display, cued the observers to the reporting location. Only one cue appeared in each trial.

External noise. External noise frames $\left(3.3^{\circ} \times 3.3^{\circ}\right)$ were made of $3 \times 3$ pixel patches $\left(0.14^{\circ} \times 0.14^{\circ}\right)$ with random contrast levels. The contrasts of the pixels in each noise frame were drawn randomly from a single Gaussian distribution with mean 0 and a certain standard deviation. To conform to the Gaussian distribution, the maximum standard deviation of external noise was kept below 0.33 of maximum achievable contrast.

Spatial layout. Four pseudocharacters were always shown on each trial. Each of them occurred in the center of a $3.3^{\circ} \times 3.3^{\circ}$ square (Figure 4). The locations of the squares were clearly marked in each trial by four black frames with the same size as the squares. The centers of the squares were displaced from the center of the display by $\pm 4.14^{\circ}$ both vertically and horizontally, equivalent to $5.85^{\circ}$ eccentricity.

\section{Design}

Cue manipulation. Cues with two different temporal relations to the stimulus frame were used: a precue with a cue-target SOA of $150 \mathrm{~ms}$, and a simultaneous cue ${ }^{3}$ with a cue-target SOA of $-33 \mathrm{~ms}$.

External noise manipulation. Eight external noise levels were used in each cue condition. The contrast of the random pixels in external noise frames corresponding to a given external noise level was drawn from a

\footnotetext{
${ }^{3}$ Because temporal summation was used to present signal and noise frames, we call this cue with SOA $=-33 \mathrm{~ms}$ the simultaneous cue because it coincided with the second set of signal frames.
} 


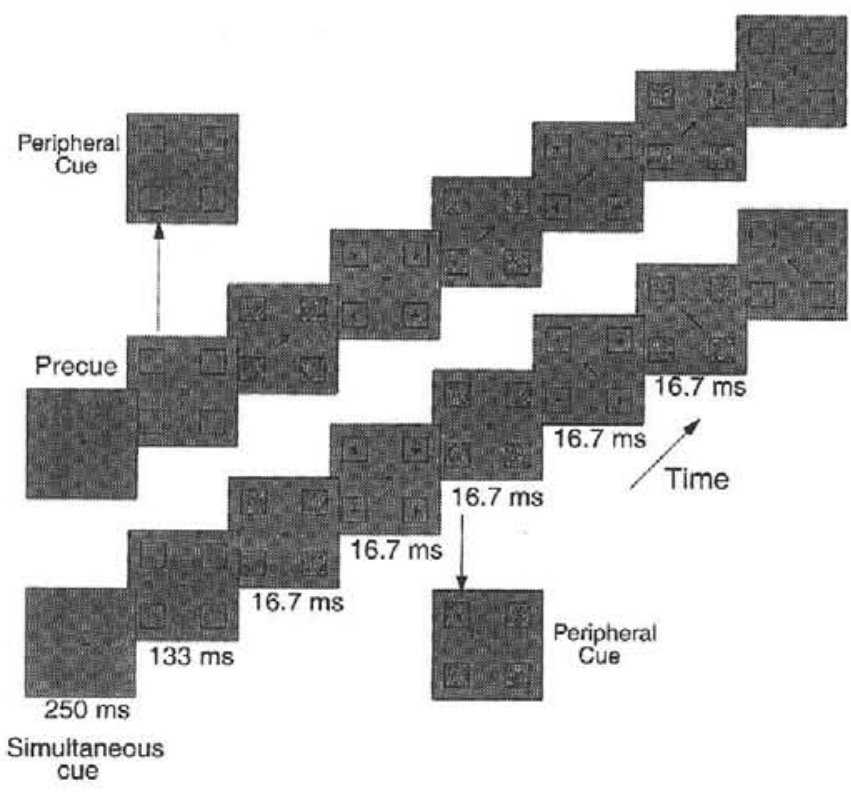

Figure 4. Procedure for Experiment 1. Following a key press, a fixation cross was presented immediately for $250 \mathrm{~ms}$. Another display with four black square outlines then came on for $133 \mathrm{~ms}$. In a precue trial, an arrow pointing to one of the four locations came on simultaneously with the four squares and stayed on throughout the entire display sequence. In a simultaneous cuc trial, the arrow cue did not appear until the presentation of the second set of target stimulus frames. Following the four-square display, five frames of $16.7 \mathrm{~ms}$ each were shown at each of the four spatial locations in the following sequence: an external noise frame, a target pseudocharacter frame, another extemal noise frame, a repetition of the target pseudochanacter frame, and an external noise frame. In the simultaneous cue condition, the cue came on simultaneously with the second signal frame. All the noise frames contained independent samples from the same Gaussian distribution. The contrast of the psendocharacters is also always the same at the four spatial locations. The observer was asked to identify the pseudocharacter at the cued location in each trial. Auditory feedback was provided after each response. In peripheral cuing (Experiment 2), the temporal sequence is identical to that of Experiment $I$. Only the spatial location of the cue is changed from the center of the display to the periphery (near the target location). This is illustrated with the two inserted examples.

Gaussian distribution with mean $\mathbf{0}$ and one of eight standard deviations: $\mathbf{0}$, $0.02,0.04,0.08,0.12,0.16,0.24$, and 0.32 . In a given trial, all the noise frames had the same level of external noise.

Contrast of pseudocharacters. Performance at nine pseudocharacter contrast levels was measured for each external noise level in each cue condition. The contrast levels were selected according to each observer's performance in the practice sessions to span a psychometric function. In a given trial, all of the four pseudocharacters had the same contrast.

\section{Procedure}

Stimulus presentation. The procedure and representative conditions of Experiment 1 are illustrated in Figure 4. The observer pressed a key to initialize a trial when ready. A fixation cross was presented immediately for $250 \mathrm{~ms}$. Another display with four black square outlines then came on for $133 \mathrm{~ms}$. The four squares always occurred in the same spatial locations and framed the subsequent stimnli. In a precue trial, an arrow pointing to one of the four locations was presented at the center of the display with the four squares and stayed on until the end of the trial. In a simultaneous cue trial, the arrow cue appeared during the target stimulus sequence and stayed on until the end of the trial. The fixation cross occupied the central part of the display prior to the cue. Following the four-square display. five blocks of two refreshes each $(16.7 \mathrm{~ms})$ were shown at each of the four sparial locations in the following sequence: an external noise block, a target pseudocharacter block, another external noise block, a repetition of the target pseudocharacter, and an external noise block. Instead of direct summation of signal and noise in each frame of presentation, temporal summation was used to guarantee linearity in the summation process. In the simultaneous cue condition, the cue came on simultaneously with the second target block. All of the noise blocks contuined independent samples of the same Gaussian distribution. Thus, the total duration from the onset of the precue to the offset of the last signal frame was limited to $208 \mathrm{~ms}$ to avoid voluntary saccades to the target location (Halleth, 1986).

Response and feedback. Observers were required to identify the orientation of the pseudocharacter at the cued location by pressing one of four keys. A beep was given immediately following every correct response. ${ }^{4}$

Experimental sessions. Observers ran 5 practice sessions with only four external noise levels $(0.0,0.08,0.16$, and 0.32$)$ and then 10 experimental sessions. Each 1-hr session consisted of 576 trials: 4 trials for each of the 2 (cue type) $\times 8$ (extemal noise levels) $\times 9$ (pseudocharacter contrast levels) conditions. All 144 types of experimental conditions were intermixed within each session. Each observer participated in 15 hr of data collection in Experiment 1 , 2,880 practice trials, and 5,760 experimental trials.

\section{Results}

Collapsing over the four spatial locations, percentages of correct responses were tabulated for each observer, each cue condition, each magnitude of external noise, and each pseudocharacter contrast level. For each observer, 16 psychometric functions resulted, each containing 40 trials at every pseudocharacter contrast level. Figure 5 plots psychometric functions for each observer at only the low and the highest external noise levels for both the precuing and the simultaneous cuing conditions. These data are representative of the remaining conditions. At each external noise level, the proportion of correct response generally increases with increasing pseudocharacter contrast. At low external noise levels, performance in the precuing and the simultaneous cuing conditions was very similar, that is, the psychometric functions in the left column of Figure 5 overlap. It is only in high external noise levels that performance in the precuing condition is much better than that in the simultaneous cuing condition, that is, the psychometric functions in the precuing conditions are above the psychometric functions in the simultaneous conditions at the same external noise level. This is

\footnotetext{
${ }^{4}$ For observers AT, QL, and SM, one beep was given for a correct response in the simultaneous cue condition; two beeps were given for a correct response in the precue condition. The following scoring system was used to encourage the observers to pay attention to the cues: five points for each correct response in the precue condition; one for each correct response in the simultaneous cue condition; zero for an incorrect response. Observers were informed of their score on each trial via auditory feedback. The accumulative score was also presented to the observer in the end of each experimental session. Referees of an earlier version of this article suggested this feedback system might have introduced a "vigilance" confound in the design. In running subsequent observers $\mathrm{AC}$ and $\mathrm{VL}$ in this experiment and all the observers in Experiment 2, only one beep was given immediately following each correct response, independent of the cue type. These two different feedback conditions did not introduce any apparent difference in the results (see below).
} 


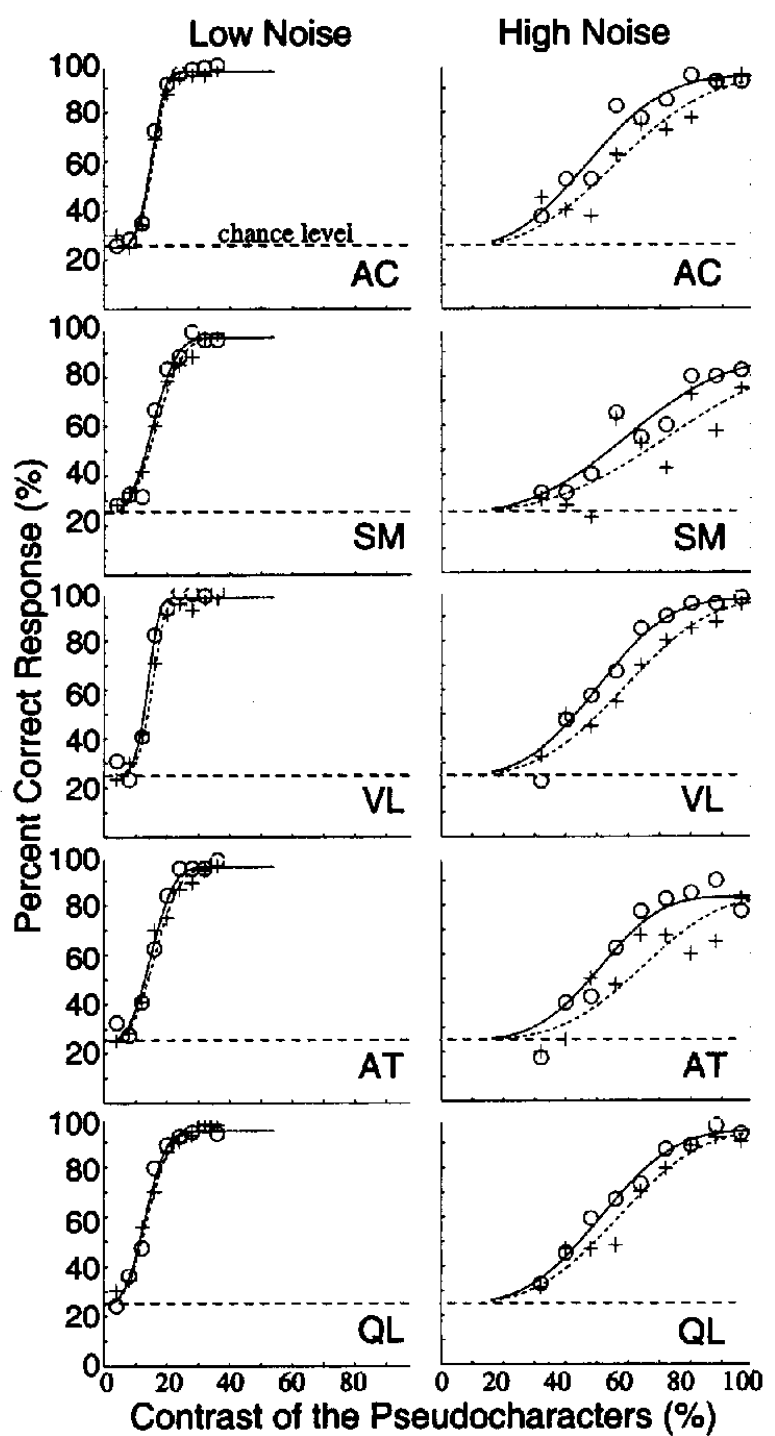

Figure S. Psychometric functions for the 5 observers in Experiment 1 in the low noise and the highest noise conditions are shown for both simultaneous cuing and precuing. A total of 16 psychometric functions were measured for each participant: 2 precuing and simultaneous cuing conditions $\times 8$ extemal noise levels. Only a subset of representative psychometric functions is shown. Smooth curves are best Weibull fits to the data. Circles and solid curves denote the precuing condition. Crosses and dotted curves denote the simultaneous cuing condition. In the low external noise condition, the two psychometric functions overlap substantially. In the high noise condition, the pair of psychometric functions in each panel differ significantly. The psychometric functions in the low noise condition are the average of those in the lowest three external noise conditions.

consistent with Lyon (1990), who observed a large precuing advantage in the presence of high contrast masks.

To quantify the difference between the precuing and the simultaneous cuing conditions, Weibull functions, ${ }^{3}$

$$
\text { Percent correct }=0.25+(\max -0.25) \times\left(1-2^{-(d / \alpha)}\right),
$$

were fit to the psychometric functions by using a maximumlikelihood ratio procedure (Hays, 1981), where $c$ is the contrast of the pseudocharacters and $\max , \alpha$, and $\eta$ are fitted parameters, assuming that minimum performance is at the chance guessing level (25\%). Each pair of psychometric functions, consisting of one from the precuing condition and the other from the simultaneous cuing condition at a given external noise level for an observer, was fit jointly by constraining the Weibull functions to have the same max and the same nonlinear coefficient $\eta^{6}{ }^{6}$

The quality of the Weibull fits is high for all the observers. The mean and the standard deviation of $r^{2}$ between the theoretical and the measured psychometric functions are 0.9757 and $0.0252,0.9629$ and $0.0404,0.9794$ and $0.0191,0.9656$ and 0.0442 , and 0.9755 and 0.0258 for participants $\mathrm{AC}, \mathrm{SM}, \mathrm{VL}, \mathrm{AT}$, and $\mathrm{QL}$, respectively.

Threshold contrasts were calculated from the Weibull fit in each experimental condition at three performance levels: $p=$ $50 \%, 62.5 \%$, and $75 \%$ correct:

$$
\tau_{p}=\alpha\left[-\log \left(1-\frac{p-0.25}{\max -0.25}\right) / \log (2.0)\right]^{1 / \eta} .
$$

Figure 6a plots the log threshold pseudocharacter contrast as a function of the log external noise contrast for both precuing and simultaneous cuing conditions for each observer at each of two performance criteria ( $d^{\prime}=0.84$ and 1.68). Error bars were estimated with a resampling procedure (Maloney, 1990): 64 resampled psychometric functions were generated by sampling a binomial distribution at each pseudocharacter contrast level with $p$ equal to the measured proportion of correct responses and $N$ equal to the total number of trials. Weibull functions were fit to each of the resampled psychometric functions by using the procedure described above. The standard deviations of the resulting 64 estimates provide an estimate of the variability in each of the three threshold pseudo-character contrasts. The error bars in Figure 6 indicate one standard deviation.

The TVC functions in Figure 6a all share the same properties: Whereas the threshold is virtually identical in the precuing and simultaneous cuing conditions in the low external noise region, the threshold in the precuing condition is lower than that in the

\footnotetext{
${ }^{5}$ Weibull functions were chosen as a convenient way to interpolate the measured psychometric functions for the purpose of estimating threshold contrast levels. Lu and Dosher (1999b) directly compared two different interpolation functions (i.e., Weibull and cumulative Gaussian) and concluded that the two forms of interpolation functions gave rise to equally good fits to the psychometric functions and virtually equal estimates of thresholds. They further tested whether the form of interpolation function (Weibull) would affect the results of subsequent PTM modeling by directly fitting the PTM to the psychometric functions. They found that PTM model parameters resulting from fitting the threshold contrasts estimated with Weibull interpolation functions were virtually identical to those resulting from direct fits to the full psychometric functions. For readers who are interested in modeling the data presented in this article, please e-mail zhonglin @usc.edu for the raw data.

'The purpose of fitting the Weibull functions to the measured psychometric functions is to interpolate the data to provide reliable estimates of contrast thresholds at three performance levels ( $p=50 \%, 62.5 \%$, and $75 \%$ correct). These fits provided stable high-quality descriptions of the data. The Weibull functions are widely used to describe psychometric functions and $\max$ is almost always fixed at 1.0, but the data in high external noise violated that strong constraint.
} 


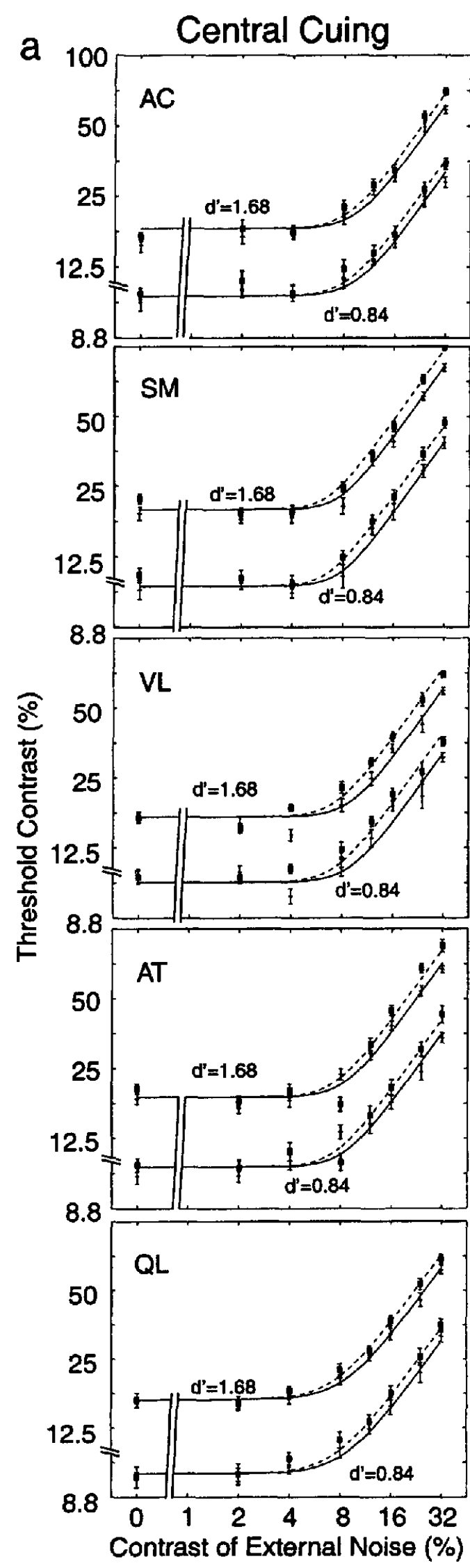

b
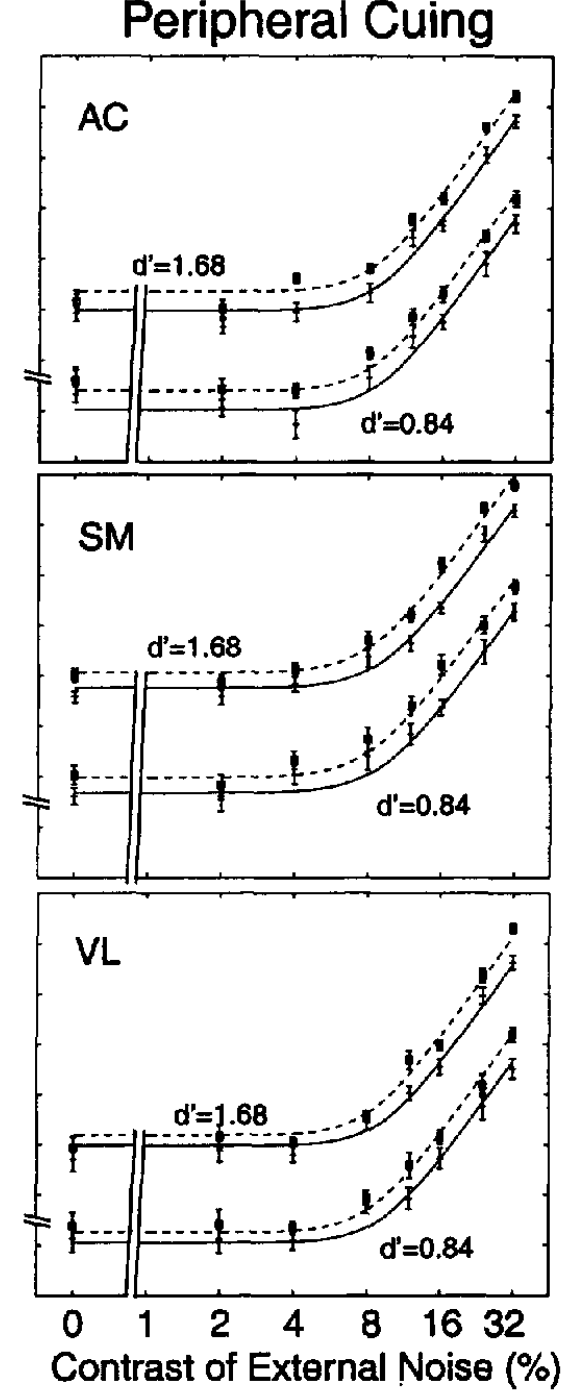

C
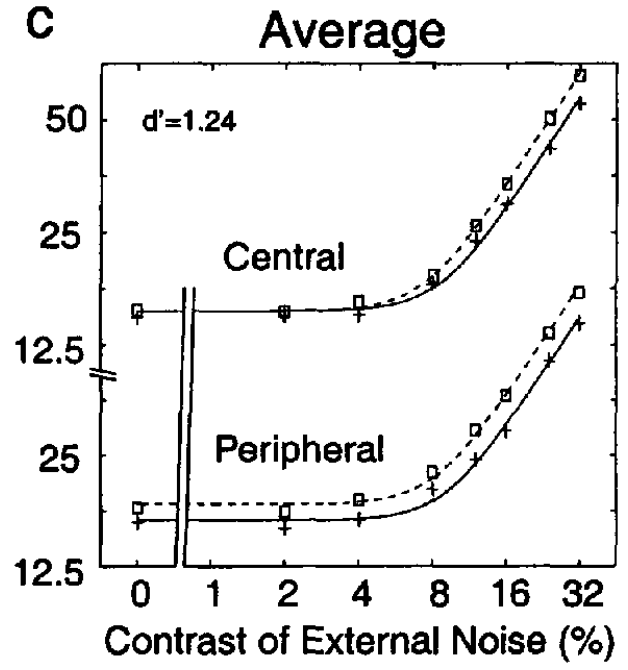
simultaneous cuing condition in the high external noise region. This pattern of results clearly suggests that external noise exclusion was the mechanism for the precuing advantage (Figures $2 \mathrm{c}$ and $2 \mathrm{~d}$ ). The size of the precuing advantage at high levels of external noise is quite substantial. It appears modest in Figure 6a because of the use of the log scale for threshold contrast. At the highest noise level, the threshold with precued spatial attention represents a $17 \%, 19 \%, 15 \%, 21 \%$, and $11 \%$ reduction for observers AC, SM, VL, AT, and QL, respectively. The data are summarized in Figure $6 \mathrm{c}$, which plots the average TVC functions at $d^{\prime}$ $=1.24$ performance level (derived by averaging the contrast threshold at $d^{\prime}=1.24$ in each condition across all the participants).

Another remarkable feature of the data shown in Figure 6a is that, for each observer, the TVC functions at two widely separated $d^{\prime}$ levels are all parallel to each other on the log scale. Comparing this feature with the theoretical predictions in Figure 3 suggests that multiplicative noise reduction was not involved in the observed central precuing advantage.

\section{Modeling}

To quantitatively characterize the attention mechanisms involved in the precuing advantage, we fit the PTM model (Equation 6) with various combinations of attention mechanisms to the external noise contrast. If there were no precuing effects, we would set $A_{\mathrm{m}}, A_{\mathrm{a}}$, and $A_{\mathrm{f}}$ to 1.0 in both the precuing and the simultaneous cuing conditions. $A$ lattice of models was generated by setting different combinations of $A$ s free to vary in the precuing condition. A total of eight models were fit and compared: (a) one model with combinations of all As; (b) three models with combinations of pairs of $A s$; (c) three models with single $A s$; and (d) one model that sets all the As to 1.0.

Programs using a gradient descent method based on Matlab 5.3 (1999) were used to find the best fitting parameters for each of the eight models. The best fitting model minimized the least squares difference between the $\log$ of the measured threshold contrast and the log of the predicted threshold. As seen in Figure 6a, the log approximately equates the standard error over a large range of contrast thresholds. Minimizing the difference in log values approximates weighted least squares, which is equivalent to the maximum-likelihood solution for continuous data. The goodness of fit is gauged by the $r^{2}$ statistic:

$$
r^{2}=1.0-\frac{\Sigma\left[\log \left(c_{\tau}^{\text {theory }}\right)-\log \left(c_{\tau}\right)\right]^{2}}{\Sigma\left[\log \left(c_{\tau}\right)-\operatorname{mean}\left(\log \left(c_{\tau}\right)\right]^{2}\right.},
$$

where $\Sigma$ and mean 0 operate over all the attention and external noise conditions at all three performance levels for a particular observer in an experiment.

Of the eight models, some are reduced models (proper subsets) of the others. An $F$ test for nested models was used to compare these models:

$$
F\left(d f_{1}, d f_{2}\right)=\frac{\left(r_{\text {full }}^{2}-r_{\text {reduced }}^{2}\right) / d f_{1}}{\left(1-r_{\text {full }}\right) / d f_{2}},
$$

where $d f_{1}=k_{\text {full }}-k_{\text {reduced, }}$ and $d f_{2}=N-k_{\text {full }}$. The $k s$ are the number of parameters in each model, and $N$ is the number of predicted data points.

Our statistical procedure identified the best model as the one with five free parameters in which attention affects only $A_{\mathrm{f}}$. This five-parameter model was superior to the four-parameter model with no precuing effects $\left(A_{\mathrm{a}}=A_{\mathrm{m}}=A_{\mathrm{f}}=1.0\right), F(1$, $43)=15.94,12.63,47.94,46.13,29.31$, all $p s<.001$, for participants AC, AT, QL, SM, and VL, respectively. No six- or seven-parameter model statistically improved the quality of fit (all ps $>.15$ ). On the other hand, dropping either $A_{\mathrm{m}}$ or $A_{\mathrm{a}}$ or both from the full model with all three attention mechanisms does not significantly decrease $r^{2}$ ( $p>.15$ ); but dropping $A_{\mathrm{f}}$ significantly decreases $r^{2}(p<.05)$. This is true for all 5 individual observers, confirming our qualitative conclusions based on the data pattern. From this, we conclude that an external noise exclusion attentional mechanism best accounts for our data on central precuing. The parameters of the best fitting model are listed in Table 1. Similar statistics resulted from fitting the average TVC functions shown in Figure 6c.

\section{Experiment 2: Peripheral Precuing With External Noise}

In this experiment, we investigated the mechanisms of attention involved in the peripheral precuing advantage. One major purpose of this experiment was to distinguish the contribution of the exogenous (vs. endogenous) attention system (Briand \& Klein, 1987; Posner, 1980; Posner \& Cohen, 1984). To facilitate the comparison of peripheral and central precuing, we used the same experimental procedure of Experiment 1 in this experiment. Only the form of cuing varied-central cues were replaced by cues in periphery. Three of the 5 observers from Experiment 1 participated in this experiment.

\section{Method}

Except where it is noted, the method is the same as that used in Experiment 1.

Figure 6. (opposite). Threshold versus external noise functions (TVC). Panel a: TVC functions for each individual participant from Experiment 1 (central cuing) at two performance levels ( $d^{\prime}=0.84$ and 1.68). Panel b: TVC functions for each individual participant from Experiment 2 (peripheral cuing) at two performance levels $\left(d^{\prime}=0.84\right.$ and 1.68). Panel c: Average TVC functions at $d^{\prime}=1.24$ across all the observers in Experiment 1 (central cuing) and Experiment 2 (peripheral cuing). Smonth curves are best fitting perceptual template model (PTM) predictions. The crosses and solid curves denote data and model predictions for the precuing condition. The squares and dotted curves denote data and model predictions for the simultaneous cuing condition. Error bars in Panels a and b are generated with a resampling method. A PTM with a single external noise exclusion mechanism of attention best fits all the data in Panel a. A PTM with a combination of external noise exclusion and stimulus enhancement mechanisms of attention best fits all the data in Panel $b$. 
Table 1

Best Fitting Perceptual Template Model Parameters (Experiment 1)

\begin{tabular}{cccccccc}
\hline Observer & $A_{\mathrm{a}}$ & $A_{\mathrm{f}}$ & $N_{\mathrm{m}}$ & $N_{\mathrm{a}}$ & $\beta$ & $\gamma$ & $r^{2}$ \\
\hline AC & 1.0 & 0.8872 & 0.0000 & 0.0041 & 1.664 & 2.224 & 0.9811 \\
SM & 1.0 & 0.8448 & 0.0000 & 0.0075 & 1.300 & 1.800 & 0.9902 \\
VL & 1.0 & 0.8190 & 0.0000 & 0.0023 & 1.534 & 2.328 & 0.9723 \\
AT & 1.0 & 0.8729 & 0.0000 & 0.0052 & 1.455 & 2.026 & 0.9745 \\
QL & 1.0 & 0.8738 & 0.0000 & 0.0106 & 0.7109 & 1.752 & 0.9932 \\
\hline
\end{tabular}

Note. $\quad A_{\mathrm{a}}=$ factor by which internal additive noise is reduced by stimulus enhancement; $A_{\mathrm{f}}=$ factor by which external noise is reduced by retuning of perceptual template (external noise exclusion); $N_{\mathrm{m}}=$ coefficient by which the standard deviation of the multiplicative internal noise is proportional to the energy in the input stimulus; $N_{\mathrm{a}}=$ standard deviation of the internal additive noise; $\beta=$ the gain of the perceptual template for the signal stimulus (assuming that it passes the external noise with gain 1.0); $\gamma=$ the exponent of the nonlinear transducer function; $r^{2}=$ a statistical measure of goodness of fit.

\section{Observers}

Three undergraduate students-AC, SM, and VL-all from the University of Southem California, participated in this experiment after finishing Experiment 1 . They were paid for their participation. Each observer performed two practice sessions (1,152 trials) before the experimental sessions.

\section{Stimuli}

Four "caret" $(L)$ cues, with corners pointing to each of the four stimulus locations, were made of black line segments (Figure 4). The corner of the caret (L) cues were $0.18^{\circ}$ vertically and $0.18^{\circ}$ horizontally away from the corner of the marking frame of the cued location. In a given trial, one of these cues, occurring next to the inner corner of the frame outlining one of the four spatial locations, cued the observer to the reporting location. Only one cue appeared in each trial.

\section{Procedure}

Stimulus presentation. The procedure and representative conditions of Experiment 2 are also illustrated in Figure 4. It is exactly analogous to that of Experiment 1.

Experimental sessions. Following their participation in Experiment 1, observers ran 2 practice sessions with only four external noise levels $(0.0,0.08,0.16$, and 0.32$)$ and then 10 experimental sessions. Each session consisted of 576 trials: 4 trials for each of the 2 (cue type) $\times 8$ (extemal noise levels) $\times 9$ (pseudocharacter contrast levels) conditions. All 144 types of experimental conditions were intermixed within each session. Each observer participated in $12 \mathrm{hr}$ of data collection. This corresponded to 5,760 experimental trials per observer.

\section{Results}

As in Experiment 1, data were collapsed across the four spatial locations, and percentages of correct responses were tabulated for each observer, each cue condition, each magnitude of external noise, and each pseudocharacter contrast level. For each observer, 16 psychometric functions resulted, each containing 40 trials at every pseudocharacter contrast level. Figure 7 plots psychometric functions for each observer at only low and the highest external noise levels for both the precuing and the simultaneous cuing conditions. At each external noise level, the proportion of correct response generally increases with increasing pseudocharacter contrast. Unlike the results from Experiment 1, performance in the precuing condition is better than that in the simultaneous cuing conditions throughout the external noise levels (Figure 7); that is, psychometric functions in the precuing conditions are shifted to the left of those in the simultaneous cuing condition.

The same procedure from Experiment 1 was followed to fit Weibull functions to the psychometric functions, to calculate

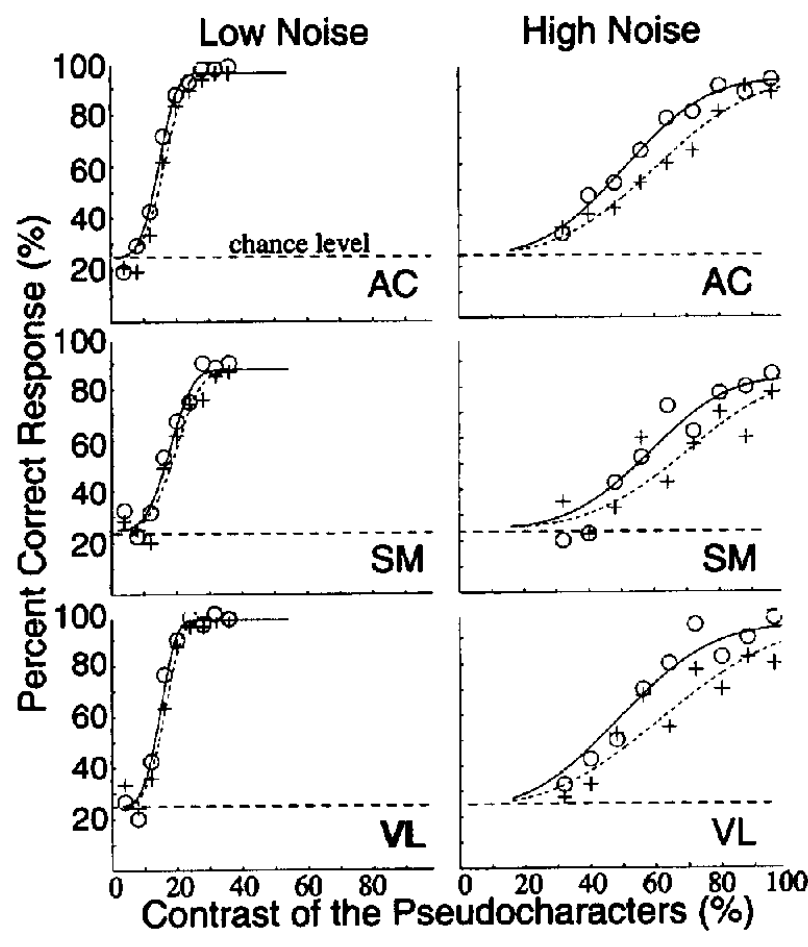

Figure 7. Psychometric functions for the 3 participants in Experiment 2 in the low noise and the highest noise conditions for both simultaneous cuing and precuing. A total of 16 psychometric functions were measured for each participant: 2 precuing and simultaneous cuing conditions $\times 8$ external noise levels. Only a subset of representative psychometric functions is shown. Smooth curves are best Weibull fits to the data. Circles and solid curves denote the precuing condition. Crosses and dotted curves denote the simultaneous cuing condition. In both low and high external noise conditions, the pair of psychometric functions in each panel differ significantly. The psychometric functions in the low noise condition are the average of those in the lowest three external noise conditions. 
threshold pseudocharacter contrasts at three performance levels: $p=50 \%, 62.5 \%$, and $75 \%$ correct, and to estimate error bars for each threshold contrast.

The quality of the Weibull fits was high for all the observers. The mean and the standard deviation of $r^{2}$ between the theoretical and the measured psychometric functions were .9777 and .0198 , .9255 and .0636 , and .9796 and .0161 for observers AC, SM, and VL, respectively.

Figure $6 \mathrm{~b}$ plots the log threshold pseudocharacter contrast as a function of the $\log$ external noise contrast for both precuing and simultaneous cuing conditions for each observer at each of the three performance criteria with error bars estimated from the resampling procedure. The data are summarized in Figure 6c, which plots the average TVC functions at $d^{\prime}=1.24$ performance level (derived by averaging the contrast threshold at $d^{\prime}=1.24$ in each condition across all the observers).

The TVC functions in Figure $6 \mathrm{~b}$ all share the same properties: Threshold at each extemal noise level in the precuing condition is lower than that in the simultaneous cuing condition at all external noise and performance levels. This pattern of results suggests a different mechanism of attention from that found in Experiment 1: A mixture of external noise exclusion and stimulus enhancement appears to be the mechanism for the precuing advantage with peripheral cues. The remarkable parallelism of all the TVC functions for each observer at three widely separated $d^{\prime}$ levels suggests that a mixture of external noise exclusion and stimulus enhancement is the mechanism for peripheral precuing advantage (see Figure 3), rather than internal multiplicative noise reduction alone or in combination with either or both of the external noise exclusion and stimulus enhancement. The parallelism of TVC functions (in the $\log$ ) is a strong, systematic property of the data, easily handled by the PTM model, which requires an explanation by any theoretical approach to the data.

The size of the precue advantage is quite substantial at all the external noise levels. Averaged across the lowest three external noise levels, the threshold with precued spatial attention represents a $13 \%, 11 \%$, and $9 \%$ threshold reduction for observers AC, SM, and VL. At the highest noise level, the threshold in the precue condition represents a $16 \%, 16 \%$, and $21 \%$ reduction for observers AC, SM, and VL, respectively. In Experiment 1, the geometric mean threshold reduction in the precue condition at the highest noise level across all the 5 observers is $16.2 \%$; the geometric mean for $\mathrm{AC}, \mathrm{SM}$, and $\mathrm{VL}$ is $16.9 \%$. Here, the geometric mean is $17.5 \%$. So, peripheral and central cuing produced essentially the same effect in the high external noise region.

\section{Modeling}

To distinguish various mixtures of attention mechanisms involved in the precue advantage in peripheral cuing, the same procedure from Experiment 1 was used to compare different model fits with the threshold data. Our statistical procedure identified the best model as the one with six free parameters in which attention affects both $A_{\mathrm{a}}$ and $A_{\mathrm{f}}-\mathrm{a}$ mixture of stimulus enhancement and external noise exclusion. This six-parameter model is superior to the four-parameter model with no precuing effects $\left(A_{\mathrm{a}}=A_{\mathrm{m}}=\right.$ $\left.A_{\mathrm{f}}=1.0\right), F(2,42)=30.63,45.37,38.37$, all $p \mathrm{~s}<.001$, for observers AC, SM, and VL. It is also superior to either fiveparameter model with stimulus enhancement only $\left(A_{\mathrm{m}}=\right.$ $\left.A_{\mathrm{f}}=1.0\right), F(1,42)=34.97,61.97,59.34$, all $p s<.001$, for observers AC, SM, and VL, or with external noise exclusion only $\left(A_{\mathrm{a}}=A_{\mathrm{m}}=1.0\right), F(1,42)=16.02,13.36,7.69, p<.01$, for observers AC, SM, and VL. On the other hand, eliminating $A_{\mathrm{m}}$ from the full model with all three mechanisms of attention does not significantly decrease $r^{2}$ (all $p s>.25$ ); but eliminating either $A_{\mathrm{f}}$ ( $p<.001$ for all 3 observers) or $A_{\mathrm{a}}(p<.025$ for AC, SM, and VL) or both ( $p<.001$ for all 3 observers) significantly decreases $r^{2}$. Similar statistical results were obtained for the average TVC functions in Figure $6 \mathrm{c}$. Thus, we conclude that a combination of stimulus enhancement and external noise exclusion mechanisms of attention operate in the peripheral cue condition. The parameters of the best fitting model are listed in Table 2 .

The geometric mean of $A_{\mathrm{f}} \mathrm{s}$ across observers AC, SM, and VL from Table 2 is .8233 . In comparison, the geometric mean of $A_{\mathrm{f}} \mathrm{s}$ across participants AC, SM, and VL in Table 1 is 0.8499 . Thus, peripheral cuing might have some very slight effects in the high noise conditions than what exists in central cuing. However, the difference is not very large. On the other hand, peripheral cuing does introduce a significant precue advantage in the low noise conditions, which did not exist with central cuing.

\section{General Discussion and Conclusions}

In this study, we applied the external noise plus attention paradigm to investigate the mechanisms of attention involved in central and peripheral precuing advantages. We found, in Experiment 1, that central precuing at 150 -ms SOA (vs. simultaneous cuing at $-33-\mathrm{ms}$ SOA) significantly improved performance (reduced threshold by an average of $16 \%$ ) only in the presence of high external noise, not in low levels of external noise. In contrast, in Experiment 2, peripheral precuing at 150-ms SOA (vs. simul-

Table 2

Best Fitting Perceptual Template Model Parameters (Experiment 2)

\begin{tabular}{cccccccc}
\hline Participant & $A_{\mathrm{a}}$ & $A_{\mathrm{f}}$ & $N_{\mathrm{m}}$ & $N_{\mathrm{a}}$ & $\beta$ & $\gamma$ & $r^{2}$ \\
\hline AC & 0.7628 & 0.8368 & 0.0000 & 0.0054 & 1.492 & 2.094 & 0.9833 \\
SM & 0.8221 & $\mathbf{0 . 8 0 4 9}$ & 0.0000 & 0.0094 & 1.203 & 1.866 & 0.9865 \\
VL & 0.8575 & 0.8284 & 0.0000 & 0.0046 & 1.565 & 2.154 & 0.9889 \\
\hline
\end{tabular}

Note. $A_{\mathrm{a}}=$ factor by which internal additive noise is reduced by stimulus enhancement; $A_{\mathrm{f}}=$ factor by which external noise is reduced by retuning of perceptual template (external noise exclusion); $N_{\mathrm{m}}=$ coefficient by which the standard deviation of the multiplicative internal noise is proportional to the energy in the input stimulus; $N_{\mathrm{a}}=$ standard deviation of the internal additive noise; $\beta=$ the gain of the perceptual template for the signal stimulus (assuming that it passes the external noise with gain 1.0); $\gamma=$ the exponent of the nonlinear transducer function; $r^{2}=$ a statistical measure of goodness of fit. 
taneous cuing at $-33-\mathrm{ms}$ SOA) significantly improved performance in the presence of high external noise (reduced threshold by an average of $17.5 \%$ ) as well as in low levels of external noise (reduced threshold by an average of $11 \%$ ). Quantitative PTM model fits found that, under the specific conditions investigated, central precuing improves performance by way of external noise exclusion associated with better tuning of the perceptual template at the precued locations; peripheral precuing improves performance by way of a combination of stimulus enhancement (or equivalently, internal additive noise reduction) and external noise exclusion.

\section{Identifying the Mechanisms}

Our results in the presence of high external noise are largely consistent with previous findings in the literature (e.g., Cheal \& Lyon, 1991; Lyon, 1990). For example, Lyon found systematic $d^{\prime}$ changes as he varied the SOA between the cue and the pseudocharacter targets in the presence of highly effective poststimulus mask. In fact, Cheal and Lyon (1992) stated that the presence of a poststimulus mask was a necessary condition for the existence of precue advantage. Because delayed masking is a form of noise, these results are plausibly related to our results on external noise exclusion. On the other hand, the external noise plus attention paradigm and the related theoretical framework enabled us, for the first time, to robustly identify the mechanisms of attention involved in central and peripheral precuing advantages in this task.

Enns and Di Lollo (1997) suggested that suppression of masking was the mechanism for the precuing advantage. If we assume that high contrast masking has effects similar to those of high external noise, our conclusion in central precuing is consistent with theirs. In peripheral cuing, we observed some effects due to stimulus enhancement in addition to external noise exclusion.

The PTM provides a general framework within which we could quantify the effects of various attention manipulations. On the other hand, the qualitative interpretation of the data patterns does not depend on the details of the PTM modeling, that is, any observer model would associate threshold reduction in the absence of external noise with stimulus enhancement and threshold reduction in high external noise with external noise exclusion.

Studying a concurrent location-cued orientation discrimination task at two locations, Lu and Dosher (1998) concluded that stimulus enhancement underlies performance improvement at the attended location. Two factors are important in comparing $\mathrm{Lu}$ and Dosher (1998) and the current results: (a) Lu and Dosher (1998) used combined central and peripheral cuing; (b) Lu and Dosher (1998) studied only two possible target locations. According to the current result, the first factor seems to suggest that both stimulus enhancement and external noise exclusion might be operative in Lu and Dosher (1998). However, we also know that external noise exclusion tends to manifest itself only when the number of possible target locations in the display is high (Cheal \& Gregory, 1997; Dosher \& Lu, 2000a; Lu \& Dosher, 2000). When there are only two possible target locations, the external noise exclusion mechanism may not be effective at all under certain display conditions (Dosher \& Lu, 2000a; Lu \& Dosher, 2000). Thus, the results from the current study are consistent with $\mathrm{Lu}$ and Dosher (1998).

The PTM is an observer model at the overall perceptual system level. A mechanism of external noise exclusion identified by the
PTM is associated with the sharpening of perceptual templates to exclude irrelevant external noise. Changes in the perceptual template refer to changes in any or all of the spatial extent, temporal tuning, or spatial frequency characteristics at the whole observer level. The exact nature of external noise exclusion is under further investigation (Lu \& Dosher, 1999a).

\section{Statistical Uncertainty Effect Versus Capacity Limitation}

In multiple-element displays, an ideal observer exhibits poorer performance in conditions where the target location is unknown than in conditions where the target location is known. This performance loss of an ideal observer is referred to as a statistical uncertainty effect. Statistical uncertainty reflects information loss and decision limits of the observer, not changes of perceptual sensitivity or limits of information-processing capacity of the ideal observer. Statistical uncertainty effects must be considered before drawing any conclusions about attention effects (Palmer, Ames, \& Lindsey, 1993; Shaw, 1984; Sperling \& Dosher, 1986).

To eliminate statistical uncertainty effects, we followed the practice of Cheal and colleagues (e.g., Cheal \& Lyon, 1992; Cheal et al., 1994; Lyon, 1990) by explicitly cuing the target location in all the cue-target SOA conditions. For an ideal observer with no capacity limitation (Palmer et al., 1993), this procedure eliminates statistical uncertainty. However, cuing cannot eliminate capacity limitations in the observer. Thus, any observed performance variation due to cue-target SOA changes in our temporal precuing paradigm reflects some form of capacity limitations of the human observers.

On the other hand, no simple limited capacity theory can account for all of our data. In the central cuing condition, we observed only a precuing advantage in the presence of high contrast external noise; in the peripheral cuing condition, we observed a smaller precuing advantage in low levels of external noise than in high levels of external noise. A simple limited capacity model such as the sample size model (Kinchla, 1980; Lindsay, Taylor, \& Forbes, 1968; Palmer, 1994; Shaw, 1980) would predict precuing advantages of the same magnitude (in terms of threshold ratio between the precuing and the simultaneous cuing conditions). This prediction is clearly falsified by the data in this study. The observed interaction between the size of the temporal precuing advantage and extemal noise level suggests a particular capacity limitation: an inability of the observer to exclude external noise simultaneously at multiple locations.

\section{Reduction of Interference From Other Locations}

Chastain and Cheal (1997) investigated the effect of distractor identity on observers' performance in a cue-target SOA experiment. They found that the accuracy in target identification increased when a large number of distractors were identical to the target. This suggested that the observers processed information from the distractor locations in spite of instructions to process information at only target locations. On the other hand, without an explicit model, the effect of distractor identity on performance is difficult to interpret. We chose a design in which the target and the distractors on every trial were all selected from the same list randomly and independently (no correlation between the identity of the pseudocharacter at the cued location and that at the uncued 
locations). Thus, in identifying the target, any cross-talk from the locations containing distractors was uncorrelated and treated as random noise.

The fact that the precue advantage occurs only in high external noise in central cuing suggests that a simple reduction of interference from other locations cannot be the only mechanism for the location precue advantage. If a simple reduction of interference from locations were the mechanism for the spatial precuing advantage, one would predict some precuing advantage over the entire range of external noise levels, because at threshold the signal-to-noise ratio, or difficulty, is the same across all the external noise conditions. This is not what we observed in central cuing.

\section{Facilitation}

Henderson (1996) reported a modest cue validity (5\% accuracy difference in two alternative forced-choice) in a paradigm in which both the target and the masking pattern appear in only one of eight locations in a given trial. In the invalid trials, if we assume that the high-intensity mask served as postcue, then there would be no spatial uncertainty about target location. Stressing that the displays had no competing locations, Henderson cited his results as supporting evidence for facilitation as the mechanism of precue advantage. However, because high contrast pattern poststimulus masks (a form of high external noise) were used in the experiment, we reinterpret his results as supporting external noise exclusion in the current theoretical framework.

Our results show that stimulus enhancement (facilitation of information acquisition at the precued location) is only significant in the peripheral cuing condition, whereas external noise exclusion is the primary mechanism for the precuing advantage in both central and peripheral cuing. Identifying stimulus enhancement (or facilitation) has not been possible previously because poststimulus masking has always been used in controlling task difficulty.

\section{Exogenous Versus Endogenous Attention}

Perhaps the most striking conclusion from the current study is that central and peripheral cuing activate different attention mechanisms. It has been proposed that two functionally separate attention systerns, an exogenous system and an endogenous system, are involved in central and peripheral cuing tasks (Briand \& Klein, 1987; Posner, 1980; Posner \& Cohen, 1984). In spatial precuing, the major observed differences between the two systems are the size (Henderson, 1991; Jonides, 1981) and time course (Cheal \& Lyon, 1991; Muller \& Rabbitt, 1989) of cuing effects. Despite their importance, the nature of these observed differences are quantitative rather than qualitative. A stronger difference in mechanism between the two attention systems is suggested by our results: external noise exclusion for the endogenous system and extemal noise exclusion plus stimulus enhancement for the exogenous system. Although this view is generally consistent with the broad literature, our results may have been a consequence of the particular experimental design. The generality of our results awaits further testing.

\section{References}

Ahumada, A. J., \& Watson, A. B. (1985). Equivalent-noise model for contrast detection and discrimination. Journal of the Optical Society of America, A, 2, 1133-1139.
Barlow, H. B. (1956). Retinal noise and absolute threshold. Joumal of the Optical Society of America, 46, 634-639.

Beck, J., \& Ambler, B. (1973). The effects of concentrated and distributed attention on peripheral acuity. Penception \& Psychophysics, 14, 225-230.

Briand, K. A., \& Klein, R. M. (1987). Is Posner's "beam" the same as Treisman's "glue"?: On the relation between visual orienting and feature integration theory. Journal of Experimental Psychology: Human Perception and Performance, 13, 228-241.

Broadbent, D. E. (1957). A mechanical model for human attention and immediate memory. Psychological Review, 64, 205-215.

Broadbent, D. E. (1971). Decision and stress. London: Academic Press.

Burgess, A. E., Shaw, R., \& Lubin, J. (Eds.). (1999, March). Noise in image processing [Special issue]. Joumal of the Optical Society of America, A, 16.

Burgess, A. E., Wagner, R. F., Jennings, R. J., \& Barlow, H. B. (1981). Efficiency of human visual signal discrimination. Science, 214, 93-94.

Chastain, G., \& Cheal, M. L. (1997). Facilitatory or inhibitory nontarget effects in the location-cuing paradigm. Consciousness \& Cognition: An International Journal, 6, 328-347.

Cheal, M. L., \& Gregory, G. (1997). Evidence of limited capacity and noise reduction with single-element displays in the location-cuing paradigm. Journal of Experimental Psychology, 23, 51-71.

Cheal, M. L., \& Lyon, D. R. (1991). Central and peripheral precuing of forced-choice discrimination. Quarterly Journal of Experimental Psy. chology: Human Experimental Psychology, 43A, 859-880.

Cheal, M. L., \& Lyon, D. R. (1992). Benefits from attention depend on the target type in location-precued discrimination. Acta Psychologica, 81, 243-267.

Cheal, M. L., Lyon, D. R., \& Gottlob, L. R. (1994). A framework for understanding the allocation of attention in location-precued discrimination. Quarterly Joumal of Experimental Psychology, 43A, 825-857.

Cheal, M. L., Lyon, D. R., \& Hubbard, D. C. (1991). Does attention have different effects on line orientation and line arrangement discrimination? Quarterly Joumal of Experimental Psychology: Human Experimental Psychology, 43A, 825-857.

Cohn, T., \& Lasley, D. J. (1974). Detectability of a luminance increment: Effect of spatial uncertainty. Journal of the Optical Society of America, 64, 1715-1719.

Corbetta, M., Miezin, F. M., Shulman, G. L., \& Petersen, S. E. (1993). A PET study of visuospatial attention. Joumal of Neuroscience, 13, 1202-1226.

Dosher, B., \& Lu, Z.-L. (1999). Mechanisms of perceptual learning. Vision Research, 39, 3197-3221.

Dosher, B., \& Lu, Z.-L. (2000a). Mechanism of perceptual attention in precuing of location. Vision Research, 40, 1269-1292.

Dosher, B., \& Lu, Z.-L. (2000b). Noise exclusion in spatial attention. Psychological Science, 11, 139-146.

Downing, C. J. (1988). Expectancy and visual-spatial attention: Effects on perceptual quality. Joumal of Experimental Psychology: Human Perception and Performance, 14, 188-202.

Egly, R., \& Homa, D. (1991). Reallocation of visual attention. Journal of Experimental Psychology: Human Perception and Performance, 17, 142-159.

Enns, J. T., \& Di Lollo, V. (1997). Object substitution: A new form of masking in unattended visual locations. Psychological Science, 8, 135-139.

Eriksen, C. W. W., \& Hoffman, J. E. (1972). Temporal and spatial characteristics of selective encoding from visual displays. Perception \& Psychophysics, 12, 201-204.

Foley, J. M. (1994). Human luminance pattern-vision mechanisms: Masking experiments require a new model. Journal of the Optical Society of America, A, 11, 1710-1719.

Foley, J. M., \& Legge, G. E. (1981). Contrast detection and near-threshold discrimination in human vision. Vision Research, 21, 1041-1053.

Fredericksen, E. R. (1996). Image manipulation and display library [computer software]. Unpublished software library. 
Grindley, G. C., \& Townsend, V. (1968). Voluntary attention in peripheral vision and its effects on acuity and differential thresholds. Quarterly Journal of Experimental Psychology, 20, 11-19.

Hallett, P. E. (1986). Eye movements. In K. R. Boff, L. Kaufman, \& J. P. Thomas (Eds.), Handbook of perception and human performance (chap. 10). New York: Wiley.

Hays, W. L. (1981). Statistics (3rd ed.). New York: CBS College Publishing.

Helmholtz, H. V. (1911). Treatise on physiological optics (3rd ed., Vols. 2 \& 3; J. P. Southall, Ed. \& Trans.). Rochester, NY: Optical Society of America. (Original work published 1866)

Henderson, J. M. (1991). Stimulus discrimination following covert attentional orienting to an exogenous cue. Journal of Experimental Psychology: Human Perception and Performance, 17, 91-106.

Henderson, J. M. (1996). Spatial precues affect target discrimination in the absence of visual noise. Joumal of Experimental Psychology: Human Perception and Performance, 22, 780-787.

Henderson, J. M., \& Macquistan, A. D. (1993). The spatial distribution of attention following an exogenous cue. Perception \& Psychophysics, 53, 221-230.

Hoffman, J. E., \& Nelson, B. (1981). Spatial selectivity in visual search. Perception \& Psychophysics, 30, 283-290.

Jonides, J. (1981). Voluntary versus automatic control over the mind's eye's movement. In J. Long \& A. Baddeley (Eds.), Attention and performance IX (pp. 187-203). Hillsdale, NJ: Erlbaum.

Jonides, J., \& Yantis, S. (1988). Uniqueness of abrupt visual onset in capturing attention. Perception \& Psychophysics, 43, 346-354.

Kinchla, R. A. (1980). The measurement of attention. In R. S. Nickerson (Ed.), Attention and performance VIII (pp. 213-238). Hillsdale, NJ: Erlbaum.

Klein, R. M., \& Hansen, E. (1990). Chronometric analysis of apparent spotlight failure in endogenous visual orienting. Journal of Experimental Psychology: Human Perception and Performance, 16, 790-801.

Lindsay, P. H., Taylor, M. M., \& Forbes, S. M. (1968). Attention and multidimensional discrimination. Perception \& Psychophysics, 4, 113-117.

Lu, Z.-L., \& Dosher, B. A. (1998). External noise distinguishes attention mechanisms. Vision Research, 38, 1183-1198.

Lu, Z.-L., \& Dosher, B. A. (1999a). Attention fine-tunes perceptual templates in spatial cuing. Bulletin of the Psychonomics Society, 40, 52.

Lu, Z.-L., \& Dosher, B. A. (1999b). Characterizing human perceptual inefficiencies with equivalent internal noise. Journal of the Optical Saciety, A, 16, 764-778.

Lu, Z.-L., \& Dosher, B. A. (2000). Effects of load in spatial attention. Manuscript in preparation.

Lu, Z.-L., Liu, C. Q., \& Dosher, B. A. (2000). Attention mechanisms for multi-location first- and second-order motion perception. Vision $R e$ search, 40, 173-186.

Lu, Z.-L., \& Sperling, G. (1999). Second-order reversed phi. Perception \& Psychophysics, 61, 1075-1088.

Lyon, D. R. (1990). Large and rapid improvement in form discrimination accuracy following a location precue. Acta Psychologica, 73, 69-82.

MacMillan, N. A., \& Creelman, C. D. (1991). Detection theory: A user's guide. New York: Cambridge University Press.

Maloney, L. T. (1990). Confidence intervals for the parameters of psychometric functions. Perception \& Psychophysics, 47, 127-134.

Mangun, G. R., Hillyard, S. A., \& Luck, S. J. (1993). Electrocortical substrates of visual selective attention. In D. E. Meyer \& S. Kornblum (Eds.), Attention and performance 14: Synergies in experimental psychology, artificial intelligence, and cognitive neuroscience (pp. 219243). Cambridge, MA: MIT Press.

Matlab 5.3 [Computer software]. (1999). Natick, MA: The Mathworks, Inc.

Muller, H. J., \& Rabbitt, P. M. (1989). Reflexive and voluntary orienting of visual attention: Time course of activation and resistance to interruption. Journal of Experimental Psychology: Human Perception and Performance, 15, 315-330.

Nagaraja, N. S. (1964). Effect of luminance noise on contrast thresholds. Joumal of the Optical Society of America, 54, 950-955.

Nakayama, K., \& Mackeben, M. (1989). Sustained and transient components of focal visual attention. Vision Research, 29, 1631-1647.

Palmer, J. (1994). Set-size effects in visual search: The effect of attention is independent of the stimulus for simple tasks. Vision Research, 34, 1703-1721.

Palmer, J., Ames, C. T., \& Lindsey, D. T. (1993). Measuring the effect of attention on simple visual search. Journal of Experimental Psychology: Human Perception and Performance, 19, 108-130.

Parish, D. H., \& Sperling, G. (1991). Object spatial frequencies, retinal spatial frequencies, noise, and the efficiency of letter discrimination. Vision Research, 31, 1399-1415.

Pelli, D. G. (1981). Effects of visual noise. Unpublished doctoral dissertation, University of Cambridge, Cambridge, England.

Pelli, D. G. (1990). The quantum efficiency of vision. In C. Blakemore (Ed.), Vision: Coding and efficiency (pp. 3-24). Cambridge, England: Cambridge University Press.

Pelli, D. G., \& Zhang, L. (1991). Accurate control of contrast on microcomputer displays. Vision Research, 31, 1337-1350.

Posner, M. I. (1980). Orienting of attention. Quarterly Journal of Experimental Psychology, 2, 3-25.

Posner, M. I., \& Cohen, Y. (1984). Components of visual orienting. In H. Bouma \& D. G. Bouwhuis (Eds.), Attention and performance X: Control of language processes (pp. 531-556). Hillsdale, NJ: Erlbaum.

Posner, M. I., Nissen, M. J., \& Ogden, W. C. (1978). Attended and unattended processing modes: The role for spatial location. In N. H. L. Pick \& I. J. Saltzman (Eds.), Modes of perceiving and processing information (pp. 137-157). Hillsdale, NJ: Erlbaum.

Posner, M. I., Snyder, R. R., \& Davidson, D. J. (1980). Attention and the detection of signals. Journal of Experimental Psychology: General, 109, 160-174.

Shaw, M. L. (1980). Identifying attentional and decision-making components in information processing. In R. S. Nickerson (Ed.), Attention and performance VIII (pp. 277-296). Hillsdale, NJ: Erlbaum.

Shaw, M. L. (1984). Division of attention among spatial locations: A fundamental difference between detection of letters and detection of luminance increments. In H. Bouma \& D. G. Bouwhuis (Eds.), Attention and performance $X$ (pp. 109-121). Hillsdale, NJ: Erlbaum.

Shiu, L., \& Pashler, H. (1994). Negligible effect of spatial precuing on identification of single digits. Journal of Experimental Psychology: Human Perception and Performance, 20, 1037-1054.

Sperling, G., \& Dosher, B. (1986). Strategy and optimization in human information processing. In K. Boff, L. Kaufman, \& J. Thomas (Eds.), Handbook of perception and performance (Vol. 1, pp. 1-85). New York: Wiley.

Sperling, G., \& Melchner, M. J. (1978). The attention operating characteristic: Examples from visual search. Science, 202, 315-318.

Wolford, G., \& Morrison, F. (1980). Processing of unattended visual information. Memory \& Cognition, 8, 521-527.

Wundt, W. (1902). Outlines of psychology (C. H. Judd, Trans.) Leipzig, Germany: W. Engelman.

Received February 25, 1999

Revision received September 13, 1999

Accepted February 9, 2000 